\title{
The correlation between cue differentiation and associative recall
}

\author{
WILLARD N. RUNQUIST and LOUISE RENNEY \\ University of Alberta, Edmonton, Alberta T6G 2E9, Canada
}

\begin{abstract}
Two experiments were conducted to correlate performance on a test of cue differentiation with cued recall. In one experiment, subjects were given $2,6,8$, or 12 study repetitions on verbal items consisting of three-word cues and single-word targets. The cues had varying numbers and patterns of identical elements. Cues were tested for differentiability and for their ability to produce target recall. In the second experiment, study repetitions on the same type of lists were followed by the two tests after the second, fourth, and final study repetitions. With a few exceptions, the frequency of occurrence of items that both were successfully differentiated and produced correct recall did not deviate significantly from predictions based on the assumption of independence of the two measures.
\end{abstract}

Interference in memory is said to occur when either the presence of other memories or concurrent processing activity reduces the accessibility of some specific trace. When traces or the cues for recall of those traces are similar to each other, interference is usually assumed to be caused by the interaction among the traces.

One view of this interaction assumes that recall occurs in relatively discrete stages (Runquist, 1975). When a cue is presented for recall, it is first encoded in some way, and then the code is used to retrieve the target. When cues leading to different memories are similar, interference may occur at either or both stages. That is, the code for one cue may be confused with the code for another, resulting in retrieval of the wrong target, or the code may directly activate several traces that block or replace the correct target.

The experiments reported here are part of a series whose thrust is to explicate the role of cue-code confusion in the production and reduction of interference. A corollary to the cue-code confusion hypothesis is that the effects of interference may be alleviated by establishing unique codes for various potentially confusable cues. That is, during the process of learning to correctly retrieve targets to specific cues, the confusability of these cues should be reduced.

While in these experiments, and many of their predecessors, the similarity among cues is physical (as opposed to semantic), we are primarily concerned with coding beyond the level of pattern recognition. The cues in these experiments consist of strings of verbal elements presented under conditions that are unlikely to result in reading errors. Thus, even though a cue is identified

Send requests for reprints to Willard Runquist, Department of Psychology, University of Alberta, Edmonton, Alberta T6G 2E9, Canada, between postal strikes. James Tousignant, Joyce Lester, Nicola Hrynyk, and Linda Butler aided in data collection. The research was supported by Grant A088 from the Natural Sciences and Engineering Research Council of Canada. correctly, the attributes of the code used to attempt retrieval may be shared with other codes and lead to interference.

This particular research was motivated by the general failure of many previous experiments to produce evidence for the establishment of discriminative cue codes during learning. The results of these experiments are generally of two kinds. In one kind of experiment, various procedures have been used to encourage or facilitate coding on the distinctive attributes of cues, but these procedures have not always resulted in the significant attenuation of interference (Nelson, Brooks, \& Fosselman, 1972; Runquist, 1973b; Runquist \& Runquist, 1978; Runquist \& Sekulich, 1979). In the second kind of experiment, independent measures of associative recall and cue-code discrimination have not revealed a strong relation between the two processes (Runquist, 1975; Runquist, Pullyblank, \& Whyte, 1982).

The experiments reported here belong to the second category. Subjects studied and were tested on pairs of verbal items in which cue elements varied in similarity. The test incorporated measures of both associative recall and cue-code discrimination. The experiments were designed to provide a detailed correlational analysis of the two measures at various levels of associative recall and with various amounts of interference present.

The testing procedure was adopted from one used in several previous experiments and was essentially the same as that used by Runquist et al. (1982). Following a study period, a series of test sequences was given. On each sequence, all but one of the cues were presented, while the subject tried to recall the correct target. Then a probe stimulus was presented. The probe was either one of the stimuli presented on the test series or the one that had been omitted. The subject was required to classify the probe as repeat (old) or missing (new) and to attempt recall of the appropriate target. 
It is assumed that during the presentation of the test set, the subject generates and stores a representation of each cue item as it is presented. When the probe is presented, it too is coded and compared with the representations of previous cues in memory. If a suitable match is found, the subject classifies it as repeated. If it is different from all the representations on memory, it is classified as missing.

The test may be distinguished from other procedures in which recall and cue recognition measures are concurrently obtained (Martin, 1967). The other procedures present list and nonlist cues on the recognition test. They require that the subject be able to distinguish list from nonlist items. On the other hand, our procedure requires the subject to distinguish which list items have been presented and which have not. To the extent that codes for the various list items are confusable, this distinction should be more difficult to make. As the subject learns to generate distinctive codes for the various list items, the effect of similarity on recognition accuracy should disappear.

The procedure for examining the correlation between cue-code discrimination and recall was similar to that used by Martin (1971) to determine the relationships between the recallability of two competing items in a retroactive inhibition paradigm. On any given probe test, the probe cue may be correctly classified (D) or not $(\bar{D})$ and may produce correct recall $(\mathrm{R})$ or not $(\overline{\mathrm{R}})$. If correct recall depends upon discriminative cue coding, then an important inequality must exist with respect to the two measures, namely, $P(D \& R)>P(R) \times P(D)$. That is, the joint probability of discrimination and recall should be greater than the product of the two separate probabilities. Were the two processes to be independent, then the two quantities would be equal. Our procedure, then, was to examine the joint occurrences of discrimination and recall in order to determine whether they deviated in the direction stated above from the equality predicted by independence.

\section{EXPERIMENT 1}

In this experiment, subjects were given $2,5,8$, or 12 study trials on paired associate lists followed by the test sequence. Lists varied in the similarity among cue terms.

\footnotetext{
Method

Materials. Paired associate lists consisted of six cue-target pairs. The cues were three unrelated CVC words, and the targets were two-syllable nouns. There were four types of lists, which varied according to the pattern of words shared among the cues for that list. In one list, the low-similarity (LS) list, 18 different words were used. For the position-constant (PC) list, six different words were in the second position, and the seventh word was in the first position for three cues and in the third position for the other three cues. The eighth word filled the remaining position in each cue. If the letters A-H stand for the eight words, the six triads would be ABC, ADC, AEC, CFA, CGA, and CHA. For the position-varied (PV) list, each triad contained a distinctive
}

word, but its position varied from cue to cue. These cues could be characterized as $\mathrm{ABC}, \mathrm{DBC}, \mathrm{BEC}, \mathrm{BFC}, \mathrm{BCG}$, and $\mathrm{BCH}$. The final list, the nonrule (NR) list, contained multiple redundancies so that there was duplication in all positions. Its pattern was $\mathrm{ABC}, \mathrm{ABD}, \mathrm{AED}, \mathrm{FGH}, \mathrm{AHC}$, and GEH. Four lists of each type were constructed using different cue and target words.

Design. The basic design was a 4 by 4 factorial design with similarity structure manipulated between subjects and number of study trials manipulated within subjects. The design required each subject to study and be tested on all four lists of the same structure. The sequence of lists was the same for all subjects in a given similarity condition. Subjects received 2 study presentations of one list, 5 presentations of another list, 8 presentations of a third list, and 12 presentations of the remaining list. The order in which these conditions occurred was counterbalanced across subjects so that each condition occurred equally often with each of the successive lists. This balancing procedure, however, confounds the ordinal position of a particular trials condition $(2,5,8,12)$ with specific lists. Subjects were 96 introductory psychology students assigned to similarity by order conditions according to a prearranged scheme that randomized the sequence of conditions within blocks containing each condition once. An additional 15 subjects were discarded for various malfunctions.

Procedure. For both study and test, items were visually presented on a TV monitor. On each list, the subject received the allotted number of study presentations followed immediately by a series of recall-recognition tests on that list. On a study trial, each word triad appeared in a linear array, with the target word displayed beneath the middle word. Items were displayed for $3 \mathrm{sec}$, and there was a $3-\mathrm{sec}$ blank at the end of the trial. Six orders of presentation of the items were used. When the study series was completed, an asterisk indicated the start of a test sequence. There were 12 consecutive test sequences, each consisting of two parts. First, five of the six triads were presented at a 3-sec rate while the subject attempted to recall. Then a question mark appeared, followed by the probe stimulus, which remained in view for $10 \mathrm{sec}$. The probe was either one of the triads that had appeared on the test sequence or the missing triad. Over the 12 sequences, each triad was omitted twice and tested once as a repeat and once as a missing item.

The subject was required to provide a confidence rating from 1 (certain the probe was repeated) to 6 (certain it was missing) and then to attempt recall of the correct target. No feedback was provided on either the paced recall sequence or to the recognition or tecall response to the probe. The sequence of trials and the order within trials was the same for all subjects.

\section{Results}

Several general findings will be reported prior to the correlational analysis. Overall levels of recall and recognition to the probe stimulus are presented in Table 1 . Significance is assumed when $\mathrm{p}<.05$.

Two aspects of the recall data are noteworthy. First, recall was as accurate for the PC list as it was for the LS list. Generally, we have found that PC lists produce some interference (Runquist \& Sekulich, 1979), but it is likely that repeating lists with the same simple rule for locating a distinctive element alleviates this interference. Second, there was little improvement in recall beyond five study presentations even when performance was considerably less than perfect. This result may be of some theoretical interest in that it implicates the role of information provided on test trials in reducing interference. Although one may only speculate at this point, feedback provided by test trials may be necessary to 
Table 1

Percent Correct Recall and Percent Correct Discrimination: Experiment 1

\begin{tabular}{|c|c|c|c|c|c|c|c|c|c|c|c|c|c|c|c|c|}
\hline \multirow[b]{2}{*}{ Similarity Structure } & & & & & \multicolumn{12}{|c|}{ Discrimination } \\
\hline & \multicolumn{4}{|c|}{ Recall } & $\mathbf{R}$ & M & Total & $\mathbf{R}$ & M & Total & $\mathbf{R}$ & $\mathbf{M}$ & Total & $\mathrm{R}$ & M & Total \\
\hline Number of Presentations & 2 & 5 & 8 & 12 & & & 2 & & & 5 & & & 8 & & & 12 \\
\hline Low Similarity & 52 & 86 & 89 & 86 & 89 & 72 & 80 & 89 & 70 & 80 & 84 & 72 & 78 & 88 & 72 & 80 \\
\hline Position Constant & 55 & 83 & 88 & 83 & 83 & 69 & 76 & 90 & 78 & 84 & 92 & 81 & 87 & 90 & 78 & 84 \\
\hline Position Varied & 30 & 59 & 58 & 63 & 83 & 58 & 70 & 85 & 59 & 72 & 85 & 64 & 75 & 94 & 57 & 75 \\
\hline Nonrule & 17 & 38 & 38 & 40 & 80 & 42 & 61 & 74 & 51 & 63 & 70 & 48 & 59 & 78 & 56 & 42 \\
\hline
\end{tabular}

Note $-R=$ repeat probes; $M=$ missing probes.

activate mechanisms leading to discriminative coding or other interference-reducing mechanisms.

While we will not present the data, two other results may be briefly mentioned. Paced recall and probe recall produced identical patterns, but paced recall was somewhat higher. Apparently, the additional demands of performing the recognition task counteracted any advantage obtained by relaxing the 3-sec time limit. Both paced and probe recall also improved over lists, but the rate of improvement did not vary for the different structures.

The results on the probe may be treated as a replicated single Latin square, thus allowing assessment of both lists and orders, even though the former is a confounded effect of position in the sequence and particular list. Some interactions among main effects are not obtainable in this procedure.

This analysis on the recall data produced main effects of structure $[F(3,80)=34.80]$, orders $[F(3,80)=$ $2.76]$, lists $[F(3,620)=51.68]$, and study trials $[F(3,620)=117.68$ and interactions of similarity structure with lists $[F(9,620)=5.17]$ and study trials $[F(9,620)=1.93]$.

The results on the recognition test presented in Table 1 are in terms of percent correct classifications. ${ }^{1}$ Confidence ratings were bimodal with over $85 \%$ of the ratings at the extremes. Consequently, the rating data will not be considered further. Although the overall picture is similar to the recall data, there are two major differences, the most important being a general lack of improvement in recognition performance with increasing amounts of study. Analysis of variance produced $F(3,620)=1.93 \quad(p=.12)$. Similarity structure produced a main effect $[F(3,80)=15.28]$, as well as interacting with repeat vs. missing (old-new) $[\mathrm{F}(3,620)=$ 4.24]. A three-way interaction of the two variables with study trials was also present $[F(9,620)=3.15]$. These interactions may be indicative of criterion effects (Underwood, 1974). In this case, subjects appear to adopt a more lenient criterion when interference is greater, with the difference increasing with greater amounts of study.

Irrespective of these complexities, whatever is learned about the cues during associative learning appears to be of little help in performing the recognition tasks, as per- formance on the latter simply does not improve with associative learning. This result is not unique to this experiment, as it has been reported previously (Nelson, Brooks, \& Wheeler, 1975; Runquist, 1975, 1978; Runquist et al., 1982).

The relationship between recall and discrimination measures was examined by comparing the frequency of probe items that both were correctly classified and produced successful recall with the frequency predicted by independence. In performing this analysis, the predicted frequency of the joint events was computed for each subject at each level of study by taking the product of the proportions of the two separate events times the number of items (12). The predicted and obtained means were then compared using $t$ tests for correlated means. Table 2 presents the results of this analysis. Shown are the mean predicted and obtained frequencies, the standard error of the difference, and $t(23)$. Obtained

Table 2

Mean Predicted and Obtained Joint Occurrences : Experiment 1

\begin{tabular}{|c|c|c|c|c|c|}
\hline & \multicolumn{5}{|c|}{ Study Trials } \\
\hline & 2 & 5 & 8 & 12 & \\
\hline \multicolumn{6}{|c|}{ Low Similarity } \\
\hline $\begin{array}{l}\text { Predicted } \\
\text { Obtained } \\
\text { SD } \\
t\end{array}$ & $\begin{array}{r}.19 \\
1.49\end{array}$ & $\begin{array}{r}5.26 \\
5.54 \\
.30 \\
1.30\end{array}$ & $\begin{array}{r}8.43 \\
8.04 \\
.09 \\
1.25\end{array}$ & $\begin{array}{r}8.71 \\
8.83 \\
.27 \\
.28\end{array}$ & $\begin{array}{l}8.78 \\
8.71\end{array}$ \\
\hline \multicolumn{6}{|c|}{ Position Constant } \\
\hline $\begin{array}{l}\text { Predicted } \\
\text { Obtained } \\
\text { SD } \\
t\end{array}$ & $\begin{array}{r}.11 \\
1.41\end{array}$ & $\begin{array}{r}5.54 \\
5.70 \\
.22 \\
.37\end{array}$ & $\begin{array}{r}8.08 \\
8.17 \\
.14 \\
.25\end{array}$ & $\begin{array}{r}8.92 \\
8.95 \\
.05 \\
.62\end{array}$ & $\begin{array}{l}9.01 \\
9.04\end{array}$ \\
\hline \multicolumn{6}{|c|}{ Position Varied } \\
\hline $\begin{array}{l}\text { Predicted } \\
\text { Obtained } \\
\text { SD } \\
t\end{array}$ & $\begin{array}{c}.13 \\
2.05^{*}\end{array}$ & $\begin{array}{r}2.11 \\
2.37 \\
.22 \\
1.57\end{array}$ & $\begin{array}{c}4.64 \\
5.00 \\
.09 \\
3.33+\end{array}$ & $\begin{array}{r}5.47 \\
5.79 \\
.16 \\
1.14\end{array}$ & $\begin{array}{l}6.82 \\
7.00\end{array}$ \\
\hline \multicolumn{6}{|c|}{ Nonrule } \\
\hline $\begin{array}{l}\text { Predicted } \\
\text { Obtained } \\
\text { SD } \\
\mathrm{t}\end{array}$ & $\begin{array}{r}.24 \\
1.32\end{array}$ & $\begin{array}{r}1.19 \\
1.50 \\
.19 \\
1.30\end{array}$ & $\begin{array}{r}2.70 \\
2.95 \\
.13 \\
1.98\end{array}$ & $\begin{array}{r}2.95 \\
3.20 \\
.14 \\
1.49\end{array}$ & $\begin{array}{l}3.37 \\
3.58\end{array}$ \\
\hline
\end{tabular}

${ }^{*} p=.05 . \quad t p=.03$. 
means consistently exceeded the predicted means, but differences were small (the largest being .39 items) and, in general, did not reach significance. Of the 16 comparisons, two achieved significance, both in the PV condition, and one fell barely short of normally acceptable levels, that in the NR condition. Whatever differences that might exist did not differ among the various similarity conditions at any level of study. Separate analyses of variance between groups at each level produced Fs less than 1.00 in every case. Since the small discrepancies between predicted and obtained frequencies did not differ among the conditions, they can most likely be attributed to small item regression effects, rather than any substantial correlation in process.

\section{EXPERIMENT 2}

The second experiment was designed to provide additional data on the relation between discrimination and recall using a different design and materials. In this experiment, subjects received two study presentations followed by the test sequence, then three study presentations followed by the test sequence, and finally, three study presentations followed by the test sequence. This design not only provides a test for the frequency of joint recalls and discriminations as in Experiment 1, but by having two test sequences separated by a study period, it is possible to consider the changes in recallability occurring from one test sequence to the next according to an item's discriminability on the first test sequence.

The theoretical issue is whether an association is more likely to be formed on a given study trial if the cue code is discriminable when the study trial begins than if the cues are not discriminated. If association formation is independent of discriminability, then the probability that an item will go from not recalled on Trial $n$ to recalled on Trial $n+1$ will not depend upon whether it was successfully discriminated on Trial $n$.

\section{Method}

The procedure was essentially the same as for Experiment 1 except for the following: (1) Strings of three consonants were used as cue terms instead of three words, (2) presentation rate was $2 \mathrm{sec}$ instead of $3 \mathrm{sec}$, (3) a new test series was initiated as soon as the subject responded to the probe (i.e., the test was subject paced instead of presented at a $10-\mathrm{sec}$ rate), (4) no practice task was used, and (5) for the recognition task, the subject responded "repeat" or "missing" and then gave a rating from one (certainty) to three (guessing). Ratings were again bimodal and will not be considered any further.

Subjects were 96 introductory psychology students assigned to one of the four similarity structure conditions as in Experiment 1. Materials were presented on IEE display cells mounted on the wall of a booth that isolated the subject and experimenter.

\section{Results}

The overall results differed somewhat from those obtained in Experiment 1. The recall and discrimination scores are presented in Table 3 in terms of percentages in order to facilitate comparisons with the previous experiment. In contrast with Experiment 1, recall in the PC condition was almost identical to the two other high-similarity groups. This result is not anomalous, however, as this level of performance is normal for these materials and conditions (Runquist, 1975). Analysis of variance of the recall data produced $F(3,92)=6.51$ for similarity and $F(2,184)=202$ for test sequence. The interaction was just below the required significance level $[F(6,184)=1.97, p=.07]$.

The major difference between the discrimination results of this experiment and those of Experiment 1 was the marked improvement over tests apparent in Experiment 2. We do not think that this result contradicts previous findings, however, since specific stimulus learning effects were confounded with general practice effects on this task. In any event, $F(3,92)=6.99$ for similarity and $F(2,184)=24.18$ for test sequences. The interaction was coincidentally identical to that for recall $[F(6,184)=1.97]$, but there was little evidence that the groups tended to converge on the third test sequence. Such convergence should be apparent if associative learning decreases the functional similarity of cue terms in the high-similarity conditions.

Table 4 presents the comparison between predicted and obtained joint frequencies of recall and discrimination. The analysis was carried out as exactly as in Experiment 1 , and the results closely resemble those obtained in that experiment. Obtained means in general exceed predicted means by a small amount. Statistically, however, only one comparison reached significance, that in the PV condition. This is also consistent with Experiment 1 . Analysis of variance of the difference scores among the similarity conditions produced only one significant $F(3,92)=5.95$, that on the third test sequence. On the other sequences, $F$ was less than 1.00 . The evidence thus seems to indicate a correlation between recall and discrimination only in the PV condition, even though the size of the effect was small.

We now turn to the changes in state resulting from the study period interpolated between test sequences. The first question to be considered is whether items that were not recalled on test Trial $n$ were recalled more frequently on Trial $n+1$ if they were correctly classified

Table 3

Percent Correct Recalls and Percent Correct Discriminations on Each Test Sequence: Experiment 2

\begin{tabular}{llccc}
\hline & & \multicolumn{3}{c}{ Test Sequence } \\
\cline { 3 - 5 } & & 1 & 2 & 3 \\
\hline \multirow{2}{*}{ Low Similarity } & Recall & 29 & 72 & 88 \\
& Discrimination & 77 & 86 & 92 \\
Position Constant & Recall & 17 & 40 & 70 \\
& Discrimination & 64 & 76 & 82 \\
Position Varied & Recall & 16 & 39 & 66 \\
& Discrimination & 70 & 73 & 79 \\
Nonrule & Recall & 24 & 46 & 66 \\
& Discrimination & 68 & 68 & 74 \\
\hline
\end{tabular}


Table 4

Mean Predicted and Obtained Joint Occurrences: Experiment 2

\begin{tabular}{|c|c|c|c|}
\hline & \multicolumn{3}{|c|}{ Test Sequence } \\
\hline & 1 & 2 & 3 \\
\hline \multicolumn{4}{|c|}{ Low Similarity } \\
\hline Predicted & 2.72 & 7.67 & 9.94 \\
\hline Obtained & 2.83 & 7.71 & 9.88 \\
\hline SD & .12 & .07 & .03 \\
\hline $\mathrm{t}$ & .90 & .65 & 2.00 \\
\hline \multicolumn{4}{|c|}{ Position Constant } \\
\hline Predicted & 1.31 & 3.85 & 7.02 \\
\hline Obtained & 1.45 & 3.96 & 7.04 \\
\hline SD & .11 & .15 & .04 \\
\hline $\mathrm{t}$ & 1.33 & .71 & .50 \\
\hline \multicolumn{4}{|c|}{ Position Varied } \\
\hline Predicted & 1.45 & 3.75 & 6.60 \\
\hline Obtained & 1.54 & 3.92 & 6.92 \\
\hline SD & .11 & .15 & .09 \\
\hline $\mathrm{t}$ & .84 & 1.16 & $3.39 *$ \\
\hline \multicolumn{4}{|c|}{ Nonrule } \\
\hline Predicted & 1.81 & 3.97 & 6.08 \\
\hline Obtained & 1.75 & 4.16 & 6.13 \\
\hline SD & .13 & .15 & .09 \\
\hline $\mathrm{t}$ & .46 & 1.29 & .54 \\
\hline
\end{tabular}

${ }^{*} p<.01$.

Table 5

Mean Proportion of Transitions From Nonrecall to Recall for Discriminated and Nondiscriminated Items

\begin{tabular}{lcccc} 
& \multicolumn{4}{c}{ Discriminability } \\
\cline { 2 - 5 } Group & Mean & SM & Mean & SM \\
\hline Low Similarity & .74 & .06 & .75 & .06 \\
Position Constant & .52 & .06 & .55 & .06 \\
Position Varied & .53 & .06 & .46 & .06 \\
Nonrule & .56 & .04 & .55 & .07 \\
\hline
\end{tabular}

on Trial $\mathrm{n}$. The analysis was conducted by determining the proportion of items recalled on Trial $n+1$ that were not recalled on Trial $n$. Separate calculations were made for those items that were correctly classified on Trial $n$ and those not correctly classified on Trial $\mathrm{n}$. The two values were obtained for each subject, and the difference between the mean proportions were tested with $t$ tests. In order to obtain a sufficient sample of items, however, it was necessary to combine the two sequence transitions. The means are presented in Table 5. Differences are small and uniformly not significant. The ts(23) were $.11, .44,1.46$, and .33 for the LS, PC, PV, and NR groups, respectively. For these data, then, associative learning appears to be independent of initial discriminability.

Before considering the theoretical implication of these results, we must deal with problems created by the fact that our conclusions represent de facto acceptance of the null hypothesis. This posture always requires the assurance that the testing procedure is powerful enough to reveal any reasonable correlation that exists. The problem is compounded by certain restrictions that exist upon data of these kinds.

It is obvious that when either recall or discrimination is zero or perfect, a difference between predicted and obtained joint events is impossible (Martin, 1971). With measures not at ceiling or floor, the maximum number of joint occurrences cannot exceed the larger frequency of the two separate events even if recall and discrimination are perfectly correlated.

We attempted to evaluate the effects of this restriction on our analysis by recomputing the ts reported in Tables 2 and 4 using the maximum obtainable joint frequency as the obtained value. The results are shown in Table 6 . The ts represent the largest value of $t$ that could have been obtained from these distributions of separate events had a perfect correlation existed between recall and discrimination. For both analyses, the maximal ts are all significant, but in a more important sense, the size of $t$ provides an estimate of the sensitivity of the analysis. The larger the value of $t$, the less correlation one should need to reach a minimal level of significance. The analysis is not equally powerful at each point, but with few exceptions, the data should be sufficiently sensitive to indicate deviations from independence of any reasonable size.

We may note in this respect that it is possible in principle to determine whether transitions from nonrecalled to recalled and nondiscriminated to discriminated were correlated. In this case, however, maximal $t$ values were well below significance at several points. This means that even with perfect correlation, significant deviations from predictions based on independence could not have been obtained.

\section{DISCUSSION}

Before concluding that recall and discrimination are indeed independent, we must deal with one other methodological problem. It is generally known that

Table 6

Maximum Obtainable t Values

\begin{tabular}{lccccccc}
\hline & \multicolumn{4}{c}{ Experiment 1 } & \multicolumn{3}{c}{ Experiment 2 } \\
\cline { 2 - 7 } & 2 & 5 & 8 & 12 & & 1 & 2 \\
\hline Low Similarity & 3.91 & 4.07 & 3.61 & 3.32 & 4.25 & 3.73 & 2.35 \\
Position Constant & 5.36 & 4.37 & 2.94 & 2.91 & 3.71 & 4.81 & 3.59 \\
Position Varied & 4.71 & 6.29 & 5.18 & 6.15 & 4.17 & 7.42 & 5.53 \\
Nonrule & 4.31 & 7.84 & 9.76 & 7.84 & 4.69 & 7.72 \\
\hline
\end{tabular}


subject abilities and item attributes that are correlated with performance on two measures may distort the relationship between the two measures when data are pooled across subjects and/or items (Runquist, 1973a). While our analyses were computed on individual subjects, hence obviating that source of variance, item effects are of some concern.

Hintzman (1980) has spelled out the possibilities in detail. Stochastic independence may result if the two measures are correlated but item attributes are negatively correlated with the individual measures, or if the correlation between the two measures is positive for some items and negative for others. We think that both possibilities are unlikely, largely because differences were relatively invariant and unsystematic across the various treatments. A theory postulating a positive contingency between recall and discrimination processes would certainly lead one to expect this component of the correlation to vary with amount of study and similarity. To attribute our results to spurious item correlations requires that the spurious correlations covary in exactly the same way. We do not wish to imply that there are no item effects, but only that the correlations are positive. Indeed, had we found the two measures to be nonindependent, a more difficult interpretative problem would have resulted. ${ }^{2}$

While the results of these experiments are reasonably clear, the theoretical implications are not nearly so. With respect to interference reduction mechanisms, the data further bolster arguments that discriminative coding of cues plays only a limited role. We again point out that it is not just the results we report here that lead to this conclusion, but a diverse set of results based on converging operations, of which stochastic independence of performance on the two tasks is simply one.

In addition, however, the results may have implications for general theories of cued retrieval. We began, as have others (e.g., Martin, 1967), by assuming that the occurrence of a nominal retrieval cue first activates some trace of its previous encoding, and this is followed by the activation of information associated with that trace. We do not think that our data necessarily invalidate this principle. A misidentification (or miscoding) of the cue could result in misrecall, but the subject may be able to identify the recalled information as incorrect and keep trying until the correct information is retrieved and recognized as such. The cues are indeed encoded in some manner, but these codes remain confusable, and the recognition test reflects this confusability.

On the other hand, it is clearly possible that a cue directly activates some memorial code involving the target item and separate codes for cues per se have not been formed during study.

There are two ways in which independence between recall and cue recognition could be accounted for. One scenario differs from that described above only in that the cue-identification phase is bypassed. According to this view (Runquist, 1974), the presentation of the cue activates correct and/or erroneous traces directly. Particular memories are then checked against whatever other information is available to determine their correctness. Again, associative interference is not eliminated. The subject achieves correct recall by learning to discriminate between correct and incorrect targets. The process is essentially a form of the generation-recognition principle (e.g., Bahrick, 1970), in which interference occurs during generation but is reduced during recognition.

We may also note that the basic principle involved is a broader version of the list-differentiation principle often used to explain reduction in retroactive and productive interference (Postman \& Underwood, 1973). Since in our task correct and "competing" targets have not been acquired in distinctively different contexts, differentiation must be made on other bases, however. It is not appropriate to speculate in detail at this time on the attributes that may be involved. Suffice it to say that variables such as frequency of cooccurrence and other variables affecting recognition (Underwood, 1974) are prime candidates.

The second possibility is that the analytic separation of cue and target information is inappropriate in that the cue directly activates some representation of the original event, which was a cue-target compound. Whether one conceives of the trace as a unitary association (Greeno, 1970) or a cue-biased encoding of the target (Tulving \& Thomson, 1973), the independence of cue recognition and associative recall exists because cue recognition tests do not activate the appropriate representations of the original events. Discriminative codes for these original events may exist and be necessary to avert interference in recall, but these codes are not utilized for performing tasks involving the cues alone.

While the above discussion does not rest entirely on the results from our version of the cue recognition test, these results have weighed heavily on our theorizing about interference phenomena. In a general sense, we have no reservations about the use of this test to index discriminative coding. The logic has been made explicit in several previous papers (Runquist, 1974; Runquist et al., 1982). It should be clear, however, that it is the fact that our test reflects the effects of cue similarity that is critical to the argument. Cue confusion is indexed by the extent to which decrements in recognition result from similarity manipulations and not by the absolute level of recognition performance, just as we infer interference in recall when similarity produces decrements in recall irrespective of the absolute level of recall. Certainly, the task demands of the recognition test (remembering whether a stimulus was presented in the current test sequence) involve processes other than cue confusability, just as recall involves processes other than interference. Conditionalizing recall on recognition, however, is tantamount to equating confusability by 
differentially eliminating items from high-similarity conditions whose recognition failure is due to that similarity.

The independence of cue recognition and recall, in our eyes, indicates that interference is primarily associative and suggests the possibility that a stage conception of cued recall may be wrong. We do not care to proceed beyond these conclusions at the present time.

We do not mean to imply that discriminative coding is never involved in associative learning, but simply that it does not normally accompany associative learning as a separate and separable process. Teaching subjects to form discriminative codes for stimuli to be used later as cues does eliminate interference (Runquist et al., 1982). Discrimination training could operate by biasing the encoding of the associative episodes in a manner enabling their later differentiation, rather than by providing separable cue codes.

Under some conditions, however, certain classes of stimuli may be encoded such that confusion is avoided. Evidence has been presented elsewhere (Runquist, 1975) that perceptually distinctive elements that require little processing of redundant elements are necessary for this to occur. This led us to expect a special status for the PC structure, in which these conditions were met (i.e., based on the rule "Process only middle element").

In the present experiment, however, it was the PV cues that appeared to generate correlations between discrimination and recall. We do not have a definitive explanation for this result. While data have been mixed with respect to the PC structure (Runquist \& Sekulich, 1979; Runquist et al., 1982), this is the first indication of any unique processes involved with PV structure. Since the correlation did not appear uniformly or consistently at various levels of study and was relatively small in magnitude, it may be wisest not to place too much importance on this one discrepancy from the general conclusion regarding independence. With respect to PC structures, however, we found absolutely no evidence that this structure should be considered especially sensitive to discriminative coding effects.

\section{REFERENCES}

B^hrick, H. P. A two phase model for prompted recall. Psychological Review, 1970, 77, 215-222.

Greeno, J, G. How associates are memorized. In D. A. Norman (Ed.), Models for memory. New York: Academic Press, 1970.

Hintzman, D. L. Simpson's paradox and the analysis of memory retrieval. Psychological Review, 1980, 87, 398-410.

Martin, E. Stimulus recognition in paired associate learning. Journal of Verbal Learning and Verbal Behavior, 1967, 6, 272. 276.
Martin, E. Verbal learning theory and independent retrieval phenomena. Psychological Review, 1971, 78, 314-332.

Nelson, D. L., Brooks, D. H., \& Wheeler, J. W. Sensory and meaning features in stimulus recognition and associative remental Psychology, 1972, 92, 305-312.

Nelson, D. L. Brooks, D. H., \& Wheeler, J. W. Sensory and meaning features in stimulus recognition and associative retrieval. Journal of Experimental Psychology: Human Learning and Memory, 1975, 1, 711-719.

Postman, L., \& Underwood, B. J. Critical issues in interference theory. Memory \& Cognition, 1973, 1, 19-40.

Runquist, W. N. Conditional recall and bias in memory experiments. American Journal of Psychology, 1973, 86, 351-359. (a)

RUnQuist, W. N. Differences in coding processes responsible for interference. Journal of Experimental Psychology, 1973, 98, 404-412. (b)

Runquist, W. N. The assessment of discriminative encoding in paired associated learning. Memory \& Cognition, 1974, 2, 472478.

Runquist, W. N. Interference among memory traces. Memory \& Cognition, 1975, 3, 143-159.

Runquist, W. N. Physical similarity and stimulus recognition: Interference could still be associative. Journal of Experimental Psychology: Human Learning and Memory, 1978, 4, 32-36.

Runquist, W. N., Pullyblank, J. C., \& Whyte, D. The differential coding of nominally similar cues and interference in recall. Journal of Experimental Psychology: Learning, Memory and Cognition, 1982, 8, 225-237.

Runquist, W. N., \& Runquist, P. A. Interference reduction with conceptually similar paired associates. Journal of Experimental Psychology: Human Learning and Memory, 1978, 4, 370-381.

Runquist, W. N., \& Sekulich, C. Transfer of stimulus differentiation and perceptual selection rules. Journal of Experimental Psychology: Human Learning and Memory, 1979, 5, 326-336.

Tulving, E., \& Thомson, D. M. Encoding specificity and retrieval processes in episodic memory. Psychological Review, 1973, 80, 352-373.

UNDE RWOOD, B. J. The role of association in recognition memory. Journal of Experimental Psychology, 1974, 102, 917-936.

\section{NOTES}

1. The recognition data may be analyzed according to the conventions of signal detection theory. Our measure, which is essentially hits plus correct rejections, is highly correlated with $d^{\prime}$ (Underwood, 1974), and analysis of $d^{\prime}$ values did not deviate from the analysis presented here.

2. One may test for independence using an overall chisquare test on the fourfold contingency table based on data combined over subjects and items. Such tests are not legitimate, however, since the entries in such tables are a priori not independent by virtue of repeated sampling from the same subjects and items. Our procedure avoids subject regression. One may note, however, that despite item and subject effects, phi coefficients in Experiment 1 , based on overall frequencies, ranged from .00 to only .32 , with a median of .15 , and were unrelated to either trials or similarity structure. Phi values for Experiment 2 ranged from -.05 to .24 , with a median of .11 , but were generally higher in the PV condition.

(Received for publication April 5, 1982; revision accepted February 9, 1983.) 